\title{
Thermophotovoltaics Bibliography
}

\section{Broman}

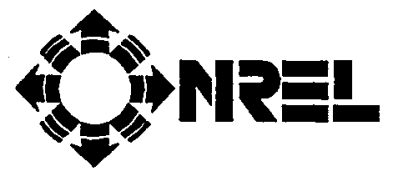

National Renewable Energy Laboratory 1617 Cole Boulevard

Golden, Colorado 80401-3393

A national laboratory of the U.S. Department of Energy Managed by Midwest Research Institute for the U.S. Department of Energy under contract No. DE-AC36-83CH10093

Prepared under Task No. PV424101 


\section{NOTICE}

This report was prepared as an account of work sponsored by an agency of the United States government. Neither the United States government nor any agency thereof, nor any of their employees, makes any warranty, express cr implied, or assumes any legal liability or responsibility for the accuracy, completeness, or usefulness of any information, apparatus, product, or process disclosed, or represents that its use would not infringe privately owned rights. Reference herein to any specific commercial product, process, or service by trade name, trademark, manufacturer, or otherwise does not necessarily constitute or imply its endorsement, recommendation, or favoring by the United States government or any agency thereof. The views and opinions of authors expressed herein do not necessarily state or reflect those of the United States government or any agency thereof.

Available to DOE and DOE contractors from:

Office of Scientific and Technical Information (OSTI)

P.O. Box 62

Oak Ridge, TN 37831

Prices available by calling (615) 576-8401

Available to the public from:

National Technical Information Service (NTIS)

U.S. Department of Commerce

5285 Port Royal Road

Springfield, VA 22161

(703) $487-4650$ 


\title{
Thermophotovoltaics Bibliography
}

\author{
Lars Broman* \\ National Renewable Energy Laboratory \\ Golden, CO 80401-3393
}

\begin{abstract}
A bibliography containing 180 entries on thermophotovoltaic conversion of energy between 1950 and 1994 has been compiled. The entries are categorized with respect to type and contents.
\end{abstract}

\section{Introduction}

Thermophotovoltaic - or TPV - conversion is the conversion of thermal radiation to electricity by means of photovoltaic (PV) cells. The thermophotovoltaic process was first suggested by Aigrain in 1960 as a two-step process for conversion of heat into electricity. The first TPV converter was built by Werth in 1963 using germanium PV cells and a propane- fueled emitter at a temperature around $1700 \mathrm{~K}$.

Since then, more than a hundred papers and reports have been published, most of them theoretical. The theoretical possibility of very high conversion efficiency has been calculated and pointed out in many papers. The optimism stemmed from the fact that crystalline silicon is transparent for photons with energies below the band-gap energy. Thus, if the back of the silicon TPV cell was coated with a highly IR-reflecting metal (e.g., silver), long-wavelength photons could be reflected back to the emitter. In 1980, Swanson reported a measured TPV conversion efficiency of $29 \%$ using silicon PVcells and a $2300 \mathrm{~K}$ graybody source.

Most of the work in the early 1980s envisioned TPV as a converter of radiation from an emitter, heated by concentrated sunlight, following the 1978 concept by Bracewell and Swanson. Many papers dealt with selective emitters or selectively reflecting filters as means to increase TPV conversion efficiency. Also other heat sources have, over the years, been discussed, such as radioactive sources for space or submarine applications. The need for very high temperatures in order to achieve good efficiency with a silicon cell-based TPV converter caused a declining interest in the process in the following years.

Presently, there is a strong renewed interest in TPV. This is due to two recent developments. One is the development of low-band-gap thin-film PV cells, originally developed for regular solar PV applications in tandem with high-band-gap cells; see Coutts and Lundstrom (1993). The other is the recent development of efficient selectively emitting fibrous emissive burners (Nelson 1992 and 1993). The first National Renewable Energy Laboratory (NREL) Conference on Thermophotovoltaic Generation of Electricity (24-27 July 1994 in Copper Mountain, Colorado) has attracted some 30 paper presentations, and the First World Conference on Photovoltaic Energy Conversion (5-9 December 1994 in Waikoloa, Hawaii) has TPV as a major topic.

This renewed interest in TPV makes this publication useful. Much research and development (R\&D) work has been done during over three decades of TPV research, and researchers of the 1990s should be able to build their research on earlier results. While many papers have been published in scientific journals or presented at international conferences, a large number were published as institute reports or patent applications, or presented at national conferences.

The present bibliography has therefore been compiled using not only current data bases, but also by searching conference proceeding contents, studying reference lists in published papers, and using personal contacts. No bibliograhy can be complete, but the author hopes that no important 
references have been missed. The reader may notice that a number of references do not specifically deal with TPV. They have been included because they contain R\&D on components or processes that can be utilized in TPV conversion and because they have been refered to in articles on TPV. When the author has not been able to get hold of a reprint, the entry says so.

In order to assist the reader, the listed articles have been categorized using the scheme below. Such a categorization is sometimes subjective, as whether a paper is a review of R\&D results or a research paper - many authors do not publish their calculations or experiments just once but many times, so when is a paper merely a review of previously published results? The entries are listed yearly, and alphabetically for each year.

The author would appreciate reader feedback. If someone knows of a reference that is missing, please send a copy of that paper or report. There is an ambition to keep up a TPV publications data base, and this should include not only this bibliography and new articles, but also older papers that are presently missing.

\section{Acknowledgements}

The good help in finding references for this bibliography by Al Burger, Mary Donahue, Kenneth Jarefors, Joergen Marks, Mats Roennelid, and Ted Wangensteen is highly appreciated. Lawrence L. Kazmerski is sincerely thanked for inviting me to NREL and being my host during my stay there.

*Centrum för solenergiforskning Solar Energy Research Center (SERC) University College of Falun Borlänge P.O. Box 10044, S-781 10 Borlänge Sweden 


\section{Categorization scheme}

[1] General/Theory/Experiment G T E

Basic research/Applied R\&D/Review/Patent/Magazine B A R P M

[2] Material/Components/System/Process M C S P

[3] Components Studied: PV-cell/Reflector/Filter/Emitter/Other P R F E O

[4] Process studied: Combustion/Other C O

[5] Energy source: Sun/Nuclear/Gas/Biomass/Fuel (other) S N G B F

[6] Application: Space/Electricity (terrestrial)/Cogeneration (terrestrial) S E C

\section{TPV References}

\section{0}

M.D. Liston and J. U. White

Amplification and electrical systems for a

double beam recording infra-red

spectrophotometer

J. Opt. Soc. Am. 40(1950)36-41

\section{2}

H. E. Kremers

Gas mantles

Encyclopedia of Chemical Technology,

Ed.s R. E. Kirk and D. F. Othmer

The Interscience Encyclopedia, Inc., N Y, 1952, Vol. 8, pp 192-197

\section{4}

R. P. Ruth and J. W. Moyer

Power efficiency for the photovoltaic

effect in a Ge grown junction

Phys. Rev. 95(1954)562-564

\section{5}

W. C. Dash and R. Newman

Intrinsic optical absorption in single crystal germanium and silicon at $77 \mathrm{~K}$ and $300 \mathrm{~K}$

Phys. Rev. 99(4/15 Aug 1955)1151-1155

\section{8}

R. Braunstein, A. R. Moore, and F. Herman Intrinsic optical absorption in GeSi alloys

Phys. Rev. 109(3/1 Feb. 1958)695-710 $\left[\begin{array}{llllll}{[1]} & {[2]} & {[3]} & {[4]} & {[5]} & {[6]}\end{array}\right.$

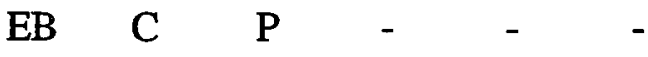

$\begin{array}{llllll} & {[2]} & {[3]} & {[4]} & {[5]} & {[6]}\end{array}$

G $\quad \mathrm{MC} \quad \mathrm{E}$

$[1] \quad[2] \quad[3] \quad[4] \quad[5] \quad[6]$

EB C $\quad P \quad$ -

$[1] \quad[2] \quad[3] \quad[4] \quad[5] \quad[6]$

EB M - $\quad$ -

$[1] \quad[2] \quad[3] \quad[4] \quad[5] \quad[6]$

EB M - $\quad$ - $\quad$ - 
B. M. Vul, V. S. Vavilov, L. S. Smirnov,

G. N. Galkin, V. M. Packevic, and

A. V. Spicyn

Ueber die Umvandlung der Energie von

b-Teilchen in Elektroenergie in

Germaniumkristallen mit pn-Uebergaengen

Kernenergie 1(4/1958)279-282

\section{9}

\section{R. Bomal}

Detection des particules nucleaires avec

les semi-conducteurs

Bull. Info. Sci. Tech. (Paris) 34(1959)2-12

\section{0}

P. Aigrain

The thermo-photovoltaic converter

Unpublished lectures given at Department

of Electrical Engineering, M. I. T. (1960/61)

\section{1}

D. C. White, B. D. Wedlock, and J. Blair

Recent advances in thermal energy conversion

Proc. 15th Ann. Power Sources Conf.

Atlantic City, NJ, May 1961, pp 125-132

\section{3}

W. L. Eisenman, R. L. Bates, and

J.D. Meriam

Black radiation detector

J. Opt. Soc. Am., 53(6/1963)729-734

R. E. Henderson

Artificially radiated energy for photovoltaic power generation

Proc. (3rd) PV Specialists Conf. (1963)

Vol. I, pp A-5-1 - A-5-23

\section{A. Thelen}

Multilayer filters with wide transmittance bands

J. Opt. Soc. Am. 53(1963)1266-1270

B. D. Wedlock

Thermal Photovoltaic Effect

Proc. (3rd) PV Specialists Conf. (1963)

Vol II, pp A-4-1 - A-4-13

B. D. Wedlock

Thermo-photo-voltaic conversion

Proc. IEEE 51, 1963, pp 694-698
$[1] \quad[2] \quad[3] \quad[4] \quad[5] \quad[6]$

EB $\quad$ C $\quad$ PV

$[1] \quad[2] \quad[3] \quad[4] \quad[5] \quad[6]$

$\begin{array}{llllll}\text { TEB } & \mathrm{C} & \mathrm{P} & - & \mathrm{N} & \mathrm{E}\end{array}$

$[1] \quad[2] \quad[3] \quad[4] \quad[5] \quad[6]$

(no reprint available)

$[1] \quad[2] \quad[3] \quad[4] \quad[5] \quad[6]$

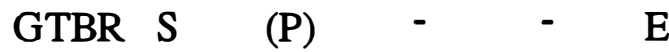

[1] [2] [3] [4] [5] [6]

EB $\quad \mathrm{C} \quad \mathrm{O} \quad \cdots \quad-$

$[1] \quad[2] \quad[3] \quad[4] \quad[5] \quad[6]$

EA (C)SP (PEO) C $\quad(\mathrm{N}) \mathrm{F} \quad(\mathrm{S}) \mathrm{E}$

$[1] \quad[2] \quad[3] \quad[4] \quad[5] \quad[6]$

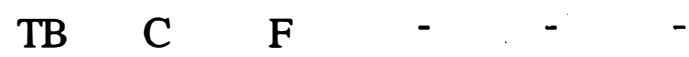

$[1] \quad[2] \quad[3] \quad[4] \quad[5] \quad[6]$

GAR (C)S (PRE) - - E

$\left[\begin{array}{llllll} & {[2]} & {[3]} & {[4]} & {[5]} & {[6]}\end{array}\right.$

TEA CS P(F) - 
J. Werth

Operation of photovoltaic cells at high power densities

Proc. (3rd) PV Specialists Conf. (1963)

Vol II, pp A-6-1 - A-6-3 (The discussion of Henderson's, Wedlock's, and Werth's presentations on pp A-6-3 - A-6-7.)

$J$. Werth

Thermophotovoltaic energy conversion

Proc. 17th Power Sources Conf.

PSC Publications Committee, Red Bank, NJ, May 1963, pp 23-27

\section{4}

A. Fortini, P. Bauduin, and P. Sibillot Realisation d'un convertisseur thermophotovoltaique

Revue Generale de l'Ectricite (Paris)

73(9/1964)466

\section{$J$. Werth}

Design study of a thermophotovoltaic converter

Proc. 18th Power Sources Conf.

PSC Publications Committee, Red Bank, NJ

May 1964, pp 153-158

D. C. White and R. L. Schwartz

P-I-N structures for controlled spectrum

PV converters

Combustion and Propulsion

Sixth Agard Colloquium

Energy Sources and Energy Conversion

Ed.s H. M. DeGroff et. al. (1964)

Gordon and Breach, N Y, pp 897-914

\section{5}

D. G. Gritton and R. C. Bourke

Radioisotope-photovoltaic energy conversion system

Adv. Energy Conversion 5(1965)119-145

B. D. Wedlock, A. Debs, R. Siegel, and G. Taylor

Investigation of germanium diodes for thermo-photovoltaic energy convertors Rpt. DA44-009-AMC-625(T) from MIT, Cambridge, MA (1965), 84 pp
$[1] \quad[2] \quad[3] \quad[4] \quad[5] \quad[6]$

EA CS PRFE - - E

$\left[\begin{array}{llllll} & {[1]} & {[3]} & {[4]} & {[5]} & {[6]}\end{array}\right.$

EBA CS PRE C G
[6]

GTA $S$

[1] [2] [3] [4] [5] [6]

EA CSP PREO $\mathrm{C}$ G

TВ $\mathrm{C} \quad \mathrm{P} \quad-\quad \cdot \quad-$

$\begin{array}{llllll}{[1]} & {[2]} & {[3]} & {[4]} & {[5]} & {[6]} \\ \text { TB } & \text { C } & P & - & - & -\end{array}$

$\left[\begin{array}{lllll}1] & {[2]} & {[3]} & {[4]} & {[5] \quad[6]}\end{array}\right.$

TA CS PFEO - N $\mathrm{N}$

[1] [2] [3] [4] [5] [6]

TEB C P - $\quad$ - 
1966

P. Bauduin and P. Sibilliot

InAs diodes used for the thermophoto-

voltaic conversion of energy

Adv. Energy Conversion 6(1966)67-70

D. P. Crouch and R. W. Beck

Study of germanium devices for use in a thermophotovoltaic converter

General Motors Corp. Progress Report

No. 2, DA28-043-AMC-01420(E)

(March 1966)

E. Kittl

Thermophotovoltaic energy conversion

Proc. 20th Power Sources Conf. (1966), pp 178-182

PSC Publ. Comm., Red Bank, NJ

B. D. Wedlock and R. Siegel

Investigation of $\mathrm{P}-\mathrm{I}-\mathrm{N}$ germanium diodes for TPV conversion

Proc. 20th Power Sources Conf. (1966), p 182

PSC Publ. Comm., Red Bank, NJ

\section{7}

R. W. Beck

Study of germanium devices for use in a thermophotovoltaic converter

General Motors Corp. Progress Report

No. 2, DA28-043-AMC-02543(E)

(Feb. 1967)

\section{S. J. Shapiro}

Thermophotovoltaic spectral analysis of a TPV system

Proc. 21st Power Sources Conf.

PSC Publ. Comm., Red Bank, NJ, 1967, pp 138-142

A. M. Vasilev

Thermophotovoltaic conversion efficiency High Temperature 5(1967)316-322

(Teplofizika Vysokikh Temperatur)

A. M. Vasilev, T. M. Golovner, A. P. Landsman, and N. S. Lidorenko Optical characteristics of silicon photocells and the efficiency of a thermophotoelectric converter

High Temperature 5(1967)967-973

(Teplofizika Vysokikh Temperatur)
[1] [2] [3] [4] [5] [6]

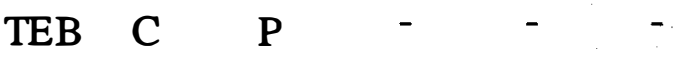

$[1] \quad[2] \quad[3] \quad[4] \quad[5] \quad[6]$

(reprint not available)

$[1] \quad[2] \quad[3] \quad[4] \quad[5] \quad[6]$

(reprint not available)

$[1] \quad[2] \quad[3] \quad[4] \quad[5] \quad[6]$

(reprint not available)
$[1] \quad[2] \quad[3] \quad[4] \quad[5] \quad[6]$

(reprint not available)

$\begin{array}{llllcc}{[1]} & {[2]} & {[3]} & {[4]} & {[5]} & {[6]} \\ \text { EB } & C & P & - & - & -\end{array}$

$\begin{array}{llllll}{[1]} & {[2]} & {[3]} & {[4]} & {[5]} & {[6]}\end{array}$

$\begin{array}{llllll}\mathrm{TB} & \mathrm{C} & \mathrm{PF} & - & (\mathrm{N}) & \text { (E) }\end{array}$

$\left[\begin{array}{llllll} & {[1]} & {[2]} & {[3]} & {[4]} & {[5]}\end{array}\right.$

TEB C(S) PR - - (E) 


\section{8}

G. Guazzoni, E. Kittl, and S. Shapiro

Status of TPV-energy conversion

Proc. Int. Electron Devices Meeting,

Washington, DC (Oct. 1968), p 128

G. Guazzoni, E. Kittl, and S. Shapiro

Rare earth radiators for thermophotovoltaic energy conversion

Proc. Int. Electron Devices Meeting,

Washington, DC (Oct. 1968), pp 130-132

\section{9}

C. W. Kim and R. J. Schwartz

A p-i-n thermo-photovoltaic diode IEEE Trans. Electron Devices 16(1969)657-663

\section{A.Thelen}

Design of multilayer interference filters

Phys. Thin Films 5(1969)47

G. E. Guazzoni

Rare-earth oxide radiators for thermophotovoltaic energy conversion Rpt. ECOM-3116 from US Army Electronics Command, Fort Monmouth, NJ (April 1969), 18 pp

\section{0}

\section{A. Kettani}

Direct Energy Conversion

Addison-Wesley, 1970, pp 416-423

\section{2}

\section{G. E. Guazzoni}

High-temperature spectral emittance of oxides of erbium, samarium, neodymium and ytterbium

Appl. Spectrosc. 26(1972)60-65

\section{G. E. Guazzoni and E. Kittl}

Experimental study of germanium cell

performance with erbium oxide and silicon carbide radiators

Rpt. ECOM-3539 from US Army

Electronics Command, Fort Monmouth, NJ (Feb. 1972), 27 pp

\section{E. Kittl and G. Guazzoni}

Design analysis of TPV-generator system Proc. 25th Power Sources Symp., May 1972, pp 106-109
$[1] \quad[2] \quad[3] \quad[4] \quad[5]$

[6]

(reprint not available)

$[1] \quad[2] \quad[3] \quad[4] \quad[5] \quad[6]$

(reprint not available) $\begin{array}{llllll}{[1]} & {[2]} & {[3]} & {[4]} & {[5]} & {[6]} \\ \text { GR } & \text { MCS } & \text { P(E) } & - & - & \text { E }\end{array}$

$[1] \quad[2] \quad[3] \quad[4] \quad[5] \quad[6]$

EB C E

$[1] \quad[2] \quad[3] \quad[4] \quad[5] \quad[6]$

EB C PE - - (E)

$[1] \quad[2] \quad[3] \quad[4] \quad[5] \quad[6]$

TA CS PFEO - $\quad F \quad$ (E) 
1973

E. Kittl

Unique correlations between blackbody radiation and optimum energy gap for a photovoltaic conversion device

E. Kittl

Energy conversion system

US Patent 3,751,303

Filed 3 June 1971, issued 7 Aug. 1973

$\begin{array}{llllll}{[1]} & {[2]} & {[3]} & {[4]} & {[5]} & {[6]} \\ \text { TB } & \text { C } & \text { P } & - & - & - \\ & & & & & \\ {[1]} & {[2]} & {[3]} & {[4]} & {[5]} & {[6]} \\ \text { GP } & \text { C } & \text { PFEO } & - & - & \text { E }\end{array}$

\section{4}

J. C. C. Fan, F. J. Bachner, G. H. Foley, and P.M. Zavracky

Transparent heat-mirror films of

$\mathrm{TiO}_{2} / \mathrm{Ag} / \mathrm{TiO}$ for solar energy collection and radiation insulation

Appl. Phys. Lett. 25(12/1974)693-695

G. E. Guazzoni and E. Kittl

Cylindrical erbium oxide radiator structures for thermophotovoltaic generator

Rpt. AD/A-001 525 from US Army

Electronics Command; distr. by NTIS,

US Dept. of Commerce (Aug. 1974), 27 pp

$\begin{array}{llllll}{[1]} & {[2]} & {[3]} & {[4]} & {[5]} & {[6]} \\ \text { EB } & \text { C } & \text { F } & - & - & -\end{array}$

$\begin{array}{llllll}{[1]} & {[2]} & {[3]} & {[4]} & {[5]} & {[6]}\end{array}$

EA MC E

\section{5}

G. E. Guazzoni and E. Kittl

Composite material structures for thermo-

photovoltaic conversion radiator

Rpt. ECOM-4351 from US Army

Electronics Command, Fort Monmouth, NJ (Sept. 1975), 22 pp

\section{6}

J. R. Yeargan, R. G. Cook, and

$F$. W. Sexton

Thermophotovoltaic systems for electrical conversion

Proc. 12th IEEE PV Specialists Conf.

(1976), pp 807-813

$\begin{array}{llllll}{[1]} & {[2]} & {[3]} & {[4]} & {[5]} & {[6]} \\ \text { EA } & \text { MC } & \text { E } & - & - & -\end{array}$

\section{7}

W. E. Horne

Solar thermal photovoltaic electric power generator

Proc. Miami Int. Conf. on Alternative

Energy Sources (1977), pp 793-795
[1]
[2]
[3]
[4]
[5]
TB
C
PR

[6]

(E)

$\begin{array}{llllll}\text { [1] } & {[2]} & {[3]} & {[4]} & {[5]} & {[6]} \\ \text { TER } & \text { CS } & \text { R(PE) } & - & S & \text { E }\end{array}$


1978

R. N. Bracewell and R. M. Swanson

Silicon photovoltaic cells in TPV

conversion

Rpt ER-633, Electric Power Res. Inst.

(February 1978), $84 \mathrm{pp}$

1979

R. L. Bell

Concentration ratio and efficiency in thermophotovoltaics

Solar Energy 23(1979)203-210

F. Demichelis and E. Minetti-Mezzetti

A solar thermophotovoltaic converter

Solar Cells 1(1979/80)395-403

R. M. Swanson

A proposed thermophotovoltaic solar energy conversion system

Proc. IEEE 67, 1979, pp 446-447

R. M. Swanson

Silicon photovoltaic cells in TPV conversion

EPRI Interim Report ER-1272, Dec. 1979

$123 \mathrm{pp}$

$\begin{array}{llllll}{[1]} & {[2]} & {[3]} & {[4]} & {[5]} & {[6]} \\ \text { TEBA CS } & \text { PR } & - & \text { S } & \text { E }\end{array}$

$[1] \quad[2] \quad[3] \quad[4] \quad[5] \quad[6]$

TB CS PF - S -

$[1] \quad[2] \quad[3] \quad[4] \quad[5] \quad[6]$

T(E)B S (F) - $\quad S \quad E$

[1] [2] [3] [4] [5] [6]

GR S - - S E

$[1] \quad[2] \quad[3] \quad[4] \quad[5] \quad[6]$

TEA $\quad \mathrm{C}(\mathrm{S}) \quad \mathrm{P}(\mathrm{R}) \quad-\quad \mathrm{S} \quad \mathrm{E}$

\section{0}

G. Castagno, F. Demichelis, and

E. Minetti-Mezzetti

Design method for multilayer interference

filters

Applied Optics 19(1980)386-388

F. Demichelis and E. Minetti-Mezzetti

Miultibandgap cells in a thermophoto-

voltaic system

Proc. 3rd EC PV Solar Energy Conference

(1980), pp 1006-1011

M. W. Edenburn

Analytical evaluation of a solar

thermophotovoltaic (TPV) converter

Solar Energy(1980)367-371

[1] [2] [3] [4] [5] [6]

TB $\mathrm{C} \quad \mathrm{F}$

[1] [2] [3] [4] [5] [6]

TEA CS PF - S E

F. C. Holden

Materials selection for the TPV radiator

Report at an EPRI Meeting (1980),

pp 6-19 - 6-27

$[1] \quad[2] \quad[3] \quad[4] \quad[5] \quad[6]$

TBA CS O - S E

$[1] \quad[2] \quad[3] \quad[4] \quad[5] \quad[6]$

GR $\quad \mathrm{MC} \quad \mathrm{E} \quad-\quad-c_{-}$ 
W. E. Horne, A. C. Day, R. B. Greegor,

L.D. Milliman, and W.L. Crabtree

Solar thermophotovoltaic space power system

Proc. 15th Intersociety Energy Conversion

Engineering Conf. (IEEE), 1980, pp 377-382

W. D. Johnston, Jr.

Solar Voltaic Cells

5.2.2 Indirect concentrators

Marcel Dekker, Inc., 1980, pp 163-166

S. Levy

Thermophotovoltaic energy conversion for electric power generation

Report at an EPRI Meeting (1980), pp 6-9 - 6-13

E. L. Paquette

Thermophotovoltaic radiator program

Report at an EPRI Meeting (1980), pp 6-29 - 6-31

R. M. Swanson

Assessment of thermophotovoltaic

conversion using silicon photovoltaic cells

Report at an EPRI Meeting (1980),

pp 6-14 - 6-15

R. M. Swanson

Recent developments in thermophoto-

voltaic conversion

Proc. 3rd EC PV Solar Energy Conference (1980), p 1097

\section{R. M. Swanson}

Recent developments in thermo-

photovoltaic conversion

Proc. Int. Electron Devices Meeting,

Washington, D. C., 8-10 Dec. 1980, pp 186-189

E. S. Vera, J. J. Loferski. M. Spitzer, and J. Schewchun

Performance of ultra high efficiency thin germanium p-n junction solar cells intended for solar thermophotovoltaic application

Proc. 3rd EC PV Solar Energy Conference (1980), pp 911-919

\section{P. Würfel and W. Ruppel}

Upper limit of thermophotovoltaic solarenergy conversion IEEE Trans. Electron Devices 27(1980)745-750
[1]
[2]
[3]
[4] [5]
[6]
TA CS
O
- $S$
$S$

$\begin{array}{llllll}{[1]} & {[2]} & {[3]} & {[4]} & {[5]} & {[6]} \\ \text { GR } & - & - & - & S & \text { (E) }\end{array}$

$[1] \quad[2] \quad[3] \quad[4] \quad[5] \quad[6]$

GR CS EP - S(N) E

$\left[\begin{array}{llllll}11 & {[2]} & {[3]} & {[4]} & {[5]} & {[6]}\end{array}\right.$

GR CS PE - S E

$\begin{array}{llllll}{[1]} & {[2]} & {[3]} & {[4]} & {[5]} & {[6]} \\ \text { GR } & \mathrm{C} & \mathrm{P} & - & - & -\end{array}$

$\left[\begin{array}{llllll} & {[1]} & {[3]} & {[4]} & {[5]} & {[6]}\end{array}\right.$

GR C P

$\begin{array}{llllcc}{[1]} & {[2]} & {[3]} & {[4]} & {[5]} & {[6]} \\ \text { EB } & C & P(R) & - & - & -\end{array}$

$\begin{array}{llllll}{[1]} & {[2]} & {[3]} & {[4]} & {[5]} & {[6]} \\ \text { TB } & \text { C } & \text { PR } & - & \text { (S) } & \text { (E) }\end{array}$

[1] [2] [3] [4] [5] [6]

TB $\mathrm{C} \quad \mathrm{FE} \quad-\quad \mathrm{S} \quad \mathrm{E}$ 


\section{1}

J. G. Severns and M. H. Cobble

A spacecraft thermophotovoltaic power source with thermal storage

Proc. 16th Intersoc. Energy Conversion

Engineering Conf. (1981), pp 89-94

E. S. Vera, J. J. Loferski, and M. Spitzer Theoretical limit efficiency of two junction tandem silicon-germanium solar cells intended for thermophotovoltaic application Proc. 15th IEEE PV Specialists Conference (1981), pp 877-882

\section{2}

M. Agnello, G. Castagno, F. Demichelis, E. Minetti-Mezzetti, and E. Tresso Research on the characteristic parameters of thermophotovoltaic (TPV) converter performance Il Nuovo Chimento 71B(1982)89-97

F. Demichelis, G. Ferrari, E. MinettiMezzetti, and W. Perotto

Refining method for the design of multilayer stacks

Applied Optics 21(10/1982)1854-1858

F. Demichelis, E. Minetti-Mezzetti, $M$. Agnello, and V. Perotto

Bandpass filters for thermophotovoltaic conversion systems

Solar Cells 5(1982)135-141

F. Demichelis, E. Minetti-Mezzetti,

$M$. Agnello, and E. Tresso

Evaluation of thermophotovoltaic efficiency

J. Appl. Phys. 53(12/1982)9098-9104

M. A. Green

Solar Cells

11.6.3 Thermophotovoltaic conversion

Prentice-Hall, Englewood Cliffs, NJ 07632 pp 217-219

W. E. Horne

Conversion of solar to electrical energy

U S Patent 4,313,024

Filed 5 April 1977, issued 26 Jan. 1982

W. E. Horne, A. C. Day, and L. Crabtree Improved thermophotovoltaic power system Proc. 17th IECEC, IEEE, 1982, pp 119-124
[1] [2] [3] [4] [5] [6]

$\begin{array}{llllll}\mathrm{TA} & \mathrm{CS} & \mathrm{PO} & (\mathrm{O}) & \mathrm{S} & \mathrm{S}\end{array}$

$[1] \quad[2] \quad[3] \quad[4] \quad[5] \quad[6]$

TA $\quad \mathrm{C} \quad \mathrm{PR} \quad-\quad(\mathrm{S})$

$[1] \quad[2] \quad[3] \quad[4] \quad[5] \quad[6]$

EB $S \quad-\quad-S \quad E$

$[1] \quad[2] \quad[3] \quad[4] \quad[5] \quad[6]$

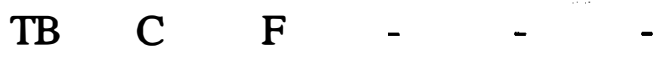

$[1] \quad[2] \quad[3] \quad[4] \quad[5] \quad[6]$

TB $\mathrm{C} \quad \mathrm{F} \quad-\quad-$

$[1] \quad[2] \quad[3] \quad[4] \quad[5] \quad[6]$

TEB $S$ - $-S$ -

$[1] \quad[2] \quad[3] \quad[4] \quad[5] \quad[6]$

$\begin{array}{lllll}\text { GR } & S \quad- & - & S & \text { (E) }\end{array}$

$[1] \quad[2] \quad[3] \quad[4] \quad[5] \quad[6]$

$\mathrm{P} \quad \mathrm{CS} \mathrm{O} \quad \mathrm{S} \quad \mathrm{E}$

[1] [2] [3] [4] [5] [6]

TA CS REO - S - 
E. S. Vera, J. J. Loferski, M. Spitzer, and J. Severns Operating characteristics of thin thermophotovoltaic cells with minority carrier mirrors and optical mirrors using selective radiators of erbium and ytterbium oxides Proc. 4th E. C. Photovoltaic Solar Energy Conference, 1982, pp 659-665

\section{3}

J. C. Bass, N. B. Elsner, and P. H. Miller Nuclear thermophotovoltaics

Proc. 18th Intersoc. Energy Conversion Engineering Conf., Vol. 5, pp 2189-2195

Am. Inst. of Ch. Engg., New York (1983)

F. Demichelis, L. Macera, E. MinettiMezzzetti, A. Tagliaferro, and E. Tresso A new apparatus to evaluate thermophotovoltaic conversion efficiency

Proc. 5th EC PV Solar Energy Conference (1983), pp 221-224

A. L. Fahrenbruch and R. H. Bube

Fundamentals of Solar Cells

Academic Press, 1983, pp 526-530

H. Höfler, H. J. Paul, W. Ruppel, and $P$. Würfel

Selective absorbers and interference filters for thermophotovoltaic energy conversion Proc. 5th EC PV Solar Energy Conference (1983), pp 225-229

H. Höfler, H. J. Paul, W. Ruppel, and $P$. Würfel

Interference filters for thermophotovoltaic solar energy conversion

Solar Cells 10(1983)273-286

H. Höfler, H. J. Paul, W. Ruppel,

Selective emitters for thermophotovoltaic solar energy conversion

Solar Cells 10(1983)257-271

\section{4}

J.L. Duomarco and L. Kaplow

Theoretical estimations of the efficiency of thermo-photovoltaic systems using high-intensity silicon solar cells Solar Energy 32(1984)33-40
$[1] \quad[2] \quad[3] \quad[4] \quad[5] \quad[6]$

TB CS RE - $S$
$[1] \quad[2] \quad[3] \quad[4] \quad[5] \quad[6]$

EA $S \quad(F)$

$[1] \quad[2] \quad[3] \quad[4] \quad[5] \quad[6]$

GR $\quad \mathrm{CS} \quad \mathrm{P} \quad \mathrm{C}^{-} \mathrm{S} \quad \mathrm{E}$

$[1] \quad[2] \quad[3] \quad[4] \quad[5] \quad[6]$

TA $\quad \mathrm{MC} \quad(\mathrm{P}) \mathrm{FE}-\quad(\mathrm{S}) \quad(\mathrm{E})$

$\left[\begin{array}{llllll} & {[1]} & {[2]} & {[3]} & {[4]} & {[5]}\end{array}\right.$

TB $\mathrm{C} \quad \mathrm{F}(\mathrm{E})$

$\left[\begin{array}{llllll} & {[1]} & {[3]} & {[4]} & {[5]} & {[6]}\end{array}\right.$

TEB CS PE $\quad-\quad S$

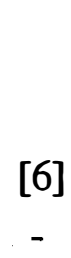

$S$ ] 
M. F. Piszczor, Jr. and M. Ghalla-Goradia Preliminary design of a $10 \mathrm{~kW}$ thermophotovoltaic system for space applications Proc. Intersoc. Energy Conv. Engg. Conf. 1984, pp 185-190

\section{5}

G. J. Addison

Yttrium oxide mantles for fuel-burning lanterns

US Patent 4,533,317

Filed 29 Aug. 1983, issued 6 Aug. 1985

\section{A. Curnu and A. M. Perroud}

Candoluminescent material and its preparation

US Patent 4,532,073

Filed 25 Feb. 1983, issued 30 July 1985

W. Spirkl and H. Ries

Solar thermophotovoltaics: An assessment J. Appl. Phys. 57(1985)4409-4414

\section{D. Woolf}

Optimum Efficiency of single and multiple band gap cells in TPV energy conversion 18th IEEE PV Specialists Conference (1985), pp 1731-1732

L. D. Woolf

Optimum efficiency of single and multiple band gap cells in TPV energy conversion Rpt. GA-A18109 from Ga Technologies (Aug. 1985), $33 \mathrm{pp}$ (preprint of Woolf's 1986 Solar Cells paper)

\section{6}

\section{R. E. Nelson}

Rare earth oxide TPV emitters

Proc. 32nd Int. Power Sources Symposium Electrochemical Society, Pennington, NJ, 1986, pp 95-101

R. E. Nelson

Thermophotovoltaic technology U S Patent 4,584,426

Filed 31 July 1984, issued 22 April 1986

C. R. Parent and R. E. Nelson

Thermophotovoltaic energy conversion with a novel rare earth oxide emitter

Proc. 21st Intersoc. Energy Conv. Engg. Conf., San Dieago, CA Am. Chem. Soc., Washington, DC, 1986 pp 1314-1317

$\begin{array}{llllll}{[1]} & {[2]} & {[3]} & {[4]} & {[5]} & {[6]} \\ \text { TA } & \text { CS } & \text { R } & - & \text { N } & \text { S }\end{array}$

[1] [2] [3] [4] [5] [6]

EP $\quad \mathrm{MC}(\mathrm{P}) \mathrm{E} \quad \mathrm{C} \quad \mathrm{F} \quad-$

$\begin{array}{llllll}{[1]} & {[2]} & {[3]} & {[4]} & {[5]} & {[6]} \\ \text { EP } & M C(P) E & C & \text { G } & -\end{array}$

[1] [2] [3] [4] [5] [6]

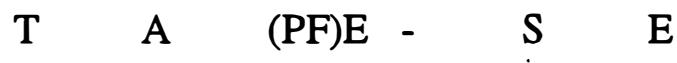

$[1] \quad[2] \quad[3] \quad[4] \quad[5] \quad[6]$

$\begin{array}{llllll}\text { TB } & \mathrm{C} & \mathrm{P} & - & - & \mathrm{E}\end{array}$

[1] [2] [3] [4] [5] [6]

TB $\mathrm{C} \quad \mathrm{P}(\mathrm{R}) \quad-\quad-\quad(\mathrm{E})$

[1] [2] [3] [4] [5] [6]

TEA C E - G -

$\left[\begin{array}{llllll}{[1]} & {[2]} & {[3]} & {[4]} & {[5]} & {[6]}\end{array}\right.$

P CS E - GF E

$[1] \quad[2] \quad[3] \quad[4] \quad[5] \quad[6]$

EBA C E - - - 
$R$. Lowe, C. Goradia, M. Goradia, and D. L. Chubb

Study of cesium plasma as a selective emitter for thermophotovoltaic applications J. Appl. Phys. 68(10/1990)5033-5035

R. E. Nelson

Thermophotovoltaic technology

US Patent 4,976,606

Filed 28 April 1989, issued 11 Dec. 1990

F. Osterle

Theoretical performance of two ultra-high efficiency photovoltaic concepts

Proc. 25th Intersoc. Energy Conversion

Conf., Reno, 1990, Vol. 5, pp 1-3

C. R. Parent

Development of more durable gas mantles

Rpt. GRI-90/0006 (PB-90-193129/XAB)

Gas Research Institute, 1990, 44 pp

$R$. Venkatasubramanian, M. L. Timmons,

R. T. Pickett, T. S. Colpitts, J.S. Hills,

J. A. Hutchby, P. A. Iles, and C. L. Chu

Development of low-bandgap $\mathrm{Ge}$ and

Si0.07Ge0.93 solar cells for monolithic and mechanically-stacked cascade applications

Proc. 21st IEEE PV Specialists Conf. 1990, pp 73-78

M. W. Wanlass, J. S. Ward, T. A. Gessert, K. A. Emery, G. S. Horner, T. J. Coutts, G. F. Virshup, and M. L. Ristow

Development of high-performance GaInAsP solar cells for tandem solar cell applications Proc. 21st IEEE PV Specialists Conference (1990), pp 172-178

\section{1}

W. G. Anderson, J. E. Lindemuth, and M.D. Morgan

Thermal management system for thermophotovoltaic/general purpose heat source power supply

Final Report DOE SBIR, Contract No.

DE-GF05-91ER81234

Thermacore Laboratory, Lancaster, PA

J.P. Edgar

Incandescent mantles

US Patent 5,071,799

Filed 28 Dec. 1989, issued 10. Dec. 1991

R. E. Nelson

Thermophotovoltaic technology

$\begin{array}{llllll}{[1]} & {[2]} & {[3]} & {[4]} & {[5]} & {[6]} \\ \text { TEB } & \text { C } & \text { E } & - & - & -\end{array}$

[1] [2] [3] [4] [5] [6]

EP MCS E

$\begin{array}{llllll}{[1]} & {[2]} & {[3]} & {[4]} & {[5]} & {[6]}\end{array}$

TB S - $\quad-S$ E

$[1]$ [2] [3] [4] [5] [6]

EA $\quad \mathrm{C}(\mathrm{P}) \quad \mathrm{E} \quad \mathrm{C} \quad \mathrm{G} \quad-$

$\begin{array}{llllll}{[1]} & {[2]} & {[3]} & {[4]} & {[5]} & {[6]}\end{array}$

EB $\quad \mathrm{MC} \quad \mathrm{P} \quad-\quad-\quad$ (E)

[1] [2] [3] [4] [5] [6]

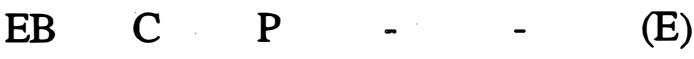

$\left[\begin{array}{llllll} & {[1]} & {[2]} & {[3]} & {[4]} & {[5]}\end{array}\right.$

(reprint not available) 
US Patent 5,057,162

Filed 31 Oct. 1990, issued 15 Oct. 1991

EP MCS E

1992

L. Block, P. Daugirda, and M. K. Goldstein Thermophotovoltaics: a new cogeneration technology for gas appliances

IEEE Trans. on Industry Applications 28(1992)251-255

T. C. Chaudhuri

A solar thermophotovoltaic converter using

$\mathrm{PbS}$ photovoltaic cells

Int. J. of Energy Research 16(1992)481-487

D. L. Chubb

Selective emitters

United States Patent No. 5,080,724,

14 January 1992, 8 pp

D. L. Chubb, D. J. Flood, and R. A. Lowe

High efficiency thermal to electric energy

conversion using selective emitters and

spectrally tuned solar cells

Rpt N92-30426 from NASA, 10 pp

D. L. Chubb, D. J. Flood, and R. A. Lowe

High efficiency thermal to electric energy

conversion using selective emitters and

spectrally tuned solar cells

Proc. Nuclear Technologies for Space

Exploration Conf., Wyoming 1992,

pp 281-293 (same as N92-30426)

J.P.Edgar

Incandescent mantles

US Patent 5,124,286

Filed 3 July 1990, issued 23 June 1992

W. E. Horne, M. D. Morgan, and A. C. Day

Radioisotope enhanced solar thermophoto-

voltaic power for the lunar surface

Solar Engineering 2(1992)861-865

J. Marks

Wood powder: an upgraded wood fuel

Forest Prod. J. 42(1992)52-56

R. E. Nelson

Fibrous emissive bumers

Selective and Broadband

Rpt. GRI-92/0347 from Gas Research Inst.

$\left[\begin{array}{llllll}{[1]} & {[2]} & {[3]} & {[4]} & {[5]} & {[6]}\end{array}\right.$

EA CS EO C $\quad$ G $\quad$ C

[1] [2] [3] [4] [5] [6]

TB CS PRFE - S $\quad$ (E)

$[1] \quad[2] \quad[3] \quad[4] \quad[5] \quad[6]$

$\mathrm{P} \quad \mathrm{C} \quad \mathrm{E} \quad-\quad-{ }-$

$\begin{array}{llllll}{[1]} & {[2]} & {[3]} & {[4]} & {[5]} & {[6]}\end{array}$

GR CS (P)E - - E

$[1] \quad[2] \quad[3] \quad[4] \quad[5] \quad[6]$

GR CS (P)E - $\quad$ - $\mathrm{E}$

Sept. 1992, 52 pp

$\begin{array}{llllll}{[1]} & {[2]} & {[3]} & {[4]} & {[5]} & {[6]} \\ P & M C & \text { E } & \text { (C) } & - & - \\ & & & & & \\ {[1]} & {[2]} & {[3]} & {[4]} & {[5]} & {[6]} \\ \text { GR } & S & - & - & \text { SN } & S \\ & & & & \cdot & \\ {[1]} & {[2]} & {[3]} & {[4]} & {[5]} & {[6]} \\ \text { GR } & \text { MCP } & \text { O } & \text { C } & \text { B } & - \\ {[1]} & {[2]} & {[3]} & {[4]} & {[5]} & {[6]} \\ \text { EA } & M C(P) & \text { E } & \text { (C) } & \text { G } & -\end{array}$


C. R. Parent, B. P. McFadden, and

J. F. S. Olow

Emission technology

US Patent 5,137,583

Filed 17 April 1991, issued 11 Aug. 1992

1993

\section{Broman and J. Marks}

TPV at NREL. Report from a visit to

[1]

[2]

[3]

[4]

[5]

[6]

National Renewable Energy Laboratory,

Golden, Colorado, U S A,

GR $\mathrm{C} \quad \mathrm{P} \quad-\quad-\quad-\quad$ E

28-29 October 1993

Rpt HFB-SERC--47--SE from SERC,

Univ. Coll. of Falun/Borlange, 6 pp

D. R. Burger and R. C. Ewell

Low-bandgap thermophotovoltaic system design

Proc. 28th Intersoc. Energy Conversion

Engg. Conf. 1993, pp 1.1045-1.1049

D. L. Chubb, D. J. Flood, and R. L. Lowe

High efficiency direct thermal to electric energy conversion from radioisotope decay using selective emitters and spectrally tuned solar cells

NASE Technical Memorandum

106227 (1993), 14 pp

D. L. Chubb and R. A. Lowe

Thin-film selective emitter

J. Appl. Phys. 74(9/1993)5687-5698

T. J. Coutts and M. S. Lundstrom

Research opportunities in crystalline III-V photovoltaics

J. Electronic Materials 22(1993)57-64

\section{A. Luque}

Coupling light to solar cells

in M. Prince, Ed., Advances in Solar

Energy, Vol. 8

ASES, Boulder, CO, 1993, pp 215-223

M. D. Morgan, W. E. Horne, and

P. R. Brothers

$\left[\begin{array}{llllll} & {[1]} & {[3]} & {[4]} & {[5]} & {[6]}\end{array}\right.$

TR (C)S (P)O - _ $\quad$ (E)

$[1] \quad[2] \quad[3] \quad[4] \quad[5] \quad[6]$

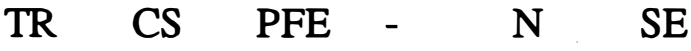

Radioisotope thermophotovoltaic power system utilizing the GaSb IR photovoltaic

cell

Proc. 10th Symp. on Space Nuclear Power and Propulsion, American Institute of

Physics (1993), pp 313-318

R. E. Nelson

Fibrous emissive burners; selective and

broad band. Annual Report

$[1] \quad[2] \quad[3] \quad[4] \quad[5] \quad[6]$

TB C E

$[1] \quad[2] \quad[3] \quad[4] \quad[5] \quad[6]$

GR $\mathrm{C} \quad \mathrm{P} \quad$ - $\quad$ -

$[1] \quad[2] \quad[3] \quad[4] \quad[5] \quad[6]$

GTR CS PF - SF E

$[1] \quad[2] \quad[3] \quad[4] \quad[5] \quad[6]$

TEA CS PO $\quad$ N $\mathrm{N}$

$\begin{array}{llllll}{[1]} & {[2]} & {[3]} & {[4] \quad[5] \quad[6]}\end{array}$

TER C E 
Aug. 1992 - July 1993

Rpt No. TR4527-029-93, GRI-93/0384 from

Gas Research Institute, $31 \mathrm{pp}$

R. E. Nelson and P. A. Iles

Possible applications of selective emitters

for space power

Proc. Joint Solar Engineering Conference, ASME 1993, pp 529-536

T. P. Ong, D. Burger, C. R. Lewis,

B. D. Campbell, and P. F. Baldasaro

Calculated performance of InAs thermo-

voltaic cells

Proc. 23rd IEEE PV Spec. Conf. (1993),

pp 705-707

\section{Valenti}

Keeping the home fires burning

Mechanical Engineering, Vol. 115,

No. 7 (July 1993), pp 66-69

$[1]$ [2] [3] [4] [5] [6]

TR C PE - SN S

M. W. Wanlass, J. S. Ward, K. A. Emery, and T.J. Coutts

Monolithic, series-connected

InP/Ga0.47In0.53As tandem solar cells

Proc. 23rd IEEE PV Specialists Conf.

(1993), pp 621-627

\section{4}

P. E. Gruenbaum, V.T. Dinh, and

V.S. Sundaram

$[1] \quad[2] \quad[3] \quad[4] \quad[5] \quad[6]$

TB $\mathrm{C} \quad \mathrm{P}$

$[1] \quad[2] \quad[3] \quad[4] \quad[5] \quad[6]$

GM S - $\quad-\quad$ G E

Gallium antimonide infrared solar cells with improved efficiency and manufacturability

Solar Energy Materials and Solar Cells 32(1994)61-69

D. M. Wilt, N. S. Fatemi, R. W. Hoffman

Jr., P. P. Jenkins, D. J. Brinker,

D. Scheiman, R. Lowe, M. Fauer, and

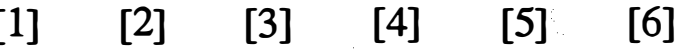

EB $\mathrm{C} \quad \mathrm{P} \quad-\quad-C_{-}$

R. K. Jain

High efficiency indium gallium arsenide photovoltaic devices for thermophotovoltaic power systems

Appl. Phys. Lett. 64(18/1994)2415-2417

$[1] \quad[2] \quad[3] \quad[4] \quad[5] \quad[6]$

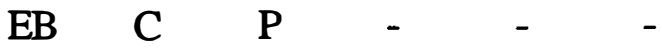

$\begin{array}{llllll}{[1]} & {[2]} & {[3]} & {[4]} & {[5]} & {[6]} \\ \text { EB } & \text { C } & P & - & - & \text { (E) }\end{array}$


Preliminary list of papers to be presented at the First NREL Conference on Thermophotovoltaic Generation of Electricity, 24-27 July 1994, Copper Mountain, Colorado

(papers not yet available for classification)

P. Adair and M. F. Rose

Composite emitters for TPV systems

R. K. Ahrenkiel, T. Wangensteen, M. M. Al-Jassim, M. Wanlass, and T. J. Coutts

Recombination lifetime of InxGal-xAs ternary alloys

P. Baldasero, E. Brown, B. Campbell, D. Depoy, and J. Parrington

Experimental assessment of low temperature voltaic energy conversion

I. Bhat, M. Ehsani, D. Morey, G. Nichols, J. Borrego, J. Parrington, and R. Gutmann

OMVPE growth and characterization of InGaAs for TPV conversion

J. Borrego and J. Parrington

Experimental assessment of low $\mathrm{T} 1$ photovoltaic energy conversion

J. Borrego, M. Zierak and G. Charache

Device modelling and parameter extraction for TPV cell development

L. Broman and J. Marks

Co-generation of electricity and heat from combustion of wood powder utilizing thermophotovoltaic conversion

D. R. Burger and R. L. Mueller

Characterization of thermophotovoltaic cells

C. Carter

The role of DOE's Division of Advanced Energy projects in thermophotovoltaics

D. L. Chubb, R. A. Lowe, and B. S. Good

Emitter theory for thin film seloctive emitter

K. Emery

Performance characterization of thermophotovoltaic cells

L.M. Fraas

Thermophotovoltaic electric generator using hydrocarbon burner

B. S. Good, D. L. Chubb, and R. A. Lowe

Temperature-dependent efficiency calculations for a thin-film selective emitter

P. E. Gruenbaum, M. S. Kuryla, and V. S. Sundaram

Technical and economic issues for gallium antimonide-based thermophotovoltaic systems

G. A. Holmquist

TPV development and the current ARPA underwater unmanned vehicle efforts

G. S. Horner, T. J. Coutts, and M. Wanlass

Proposal for a second generation lattice-matched multiple junction Ga2AsSb TPV converter

P. A. Iles

Photovoltaic principles used in thermophotovoltaic generators 
R. K. Jain, D. M. Wilt, G. A. Landis, L. Weinberg, and D. J. Flood

Low bandgap solar cells for thermophotovoltaic applications

K. Krist

GRI Research on thermophotovoltaics

R. A. Lowe, D. L. Chubb, and B. S. Good

Radiative performance of $\mathrm{Er}$ and Ho-YAG selective emitters

J. B. Milstein, R. G. Roy and D. C. Mauer

Some characteristics of a novel direct thermal-to-optical energy converter medium

R. E. Nelson

Thermophotovoltaic emitter development

E. L. Ralph and M. FitzGerald

Systems/marketing challenges for TPV

D. L. Noreen and H. Du

Porous ceramic regenerative bumer for use in lower temperature $(<1500$ (C) TPV Systems

U. C. Pernisz and C. K. Saha

Silicon carbide emitter and bumer elements for a TPV converter

R. Rosenfeld

An ARPA perspective on TPV

B. L. Sater

Vertical multi-junction cells for thermophotovoltaic conversion

A. Schock and V. Kumar

Radioisotope thermophotovoltaic system design and its application to an illustrative space mission

A. Schock, M. Mumunda, T. Or, and G. Summers

Analysis, optimization, and assessment of radioisotope thermophotovoltaic system design for an illustrative space mission

K. L. Schroeder, M. F. Rose, and J. E. Burkhalter

An experimental investigation of hybrid kerosene burner configurations for TPV applications

R. J. Schwartz and J. L. Gray

The design and modeling of photovoltaic cells for TPV systems

K. W. Stone, S. M. Kusek, and R. E. Drubka

Analysis of solar thermophotovoltaic test data from experiments performed at McDonnell Douglas

M. L. Timmons, P. R. Sharps, R. Venkatasubramanian, P. A. Iles, and C. L. Chu

Thermal photovoltaic cells

M. W. Wanlass, J. S. Ward, T. J. Coutts, and K. Emery

GaxInl-xAs thermophotovoltaic converters

J. S. Ward, X. Wu, M. S. Wanlass, and T. J. Coutts

High performance contacts to GaInAs TPV converters

S. Wojtczuk

InxGal-xAs thermophotovoltaic cell performance vs. bandgap 


\section{Other Sources}

G. H. Dieke

Spectra and energy levels of rare earth ions

$\begin{array}{lllllll} & {[1]} & {[2]} & {[3]} & {[4]} & {[5]} & {[6]} \\ \mathrm{GR} & \mathrm{M} & - & - & - & - & \end{array}$

J. A. Duffie and W. A. Beckman

Solar Engineering of Thermal Processes,

2nd Ed. (1991)

Wiley \& Sons, pp 68 and 151-153

L. Levi

Handbook of tables of functions for

$\left[\begin{array}{llllll}{[1]} & {[2]} & {[3]} & {[4]} & {[5]} & {[6]}\end{array}\right.$

applied optics

CRC Press, Cleveland OH, 1974

G - - - -

C. Blackbody radiation tables -

radiant, pp 347-409

* Permanent address: Solar Energy Research Center, University College of Falun Borlaenge, P. O. Box 10044, S-781 10 Borlaenge, SWEDEN, e-mail lbr@t.hfb.se 


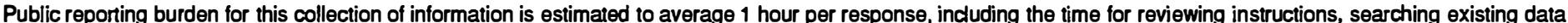

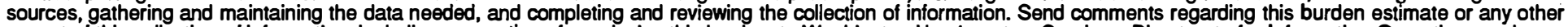

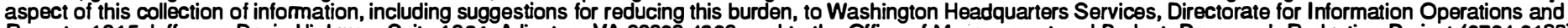

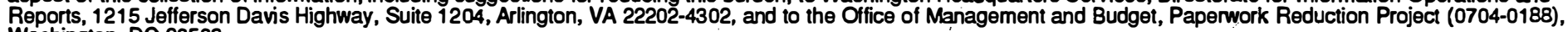
Washington, DC 20503.
1. AGENCY USE ONLY (Leave blank)
2. REPORT DATE
3. REPORT TYPE AND DATES COVERED
September 1994
Technical Report

4. TITLE AND SUBTITLE

Thermophotovoltaics Bibliography

6. AUTHOR(S)

L. Broman

7. PERFORMING ORGANIZATION NAME(S) AND ADDRESS(ES)

NREL

1617 Cole Blvd.

Golden, CO 80401
5. FUNDING NUMBERS

C:

TA: PV424101

8. PERFORMING ORGANIZATION REPORT NUMBER
9. SPONSORING/MONITORING AGENCY NAME(S) AND ADDRESS(ES)

National Renewable Energy Laboratory

1617 Cole Blvd.

Golden, CO 80401-3393
10. SPONSORING/MONITORING

AGENCY REPORT NUMBER

TP-412-6845

DE94011898

11. SUPPLEMENTARY NOTES

NREL Technical Monitor: NA

12a. DISTRIBUTION/AVAILABILITY STATEMENT

UC-1250

13. ABSTRACT (Maximum 200 words)

This is a bibliography containing 180 entries on thermophotovoltaic conversion of energy between 1950 and 1994. The entries are categorized with respect to type and contents.

\section{SUBJECT TERMS}

thermophotovoltaics ; photovoltaics ; solar cells
15. NUMBER OF PAGES

25

16. PRICE CODE
17. SECURITY CLASSIFICATION OF REPORT

Unclassified
18. SECURITY CLASSIFICATION OF THIS PAGE Unclassified
19. SECURITY CLASSIFICATION OF ABSTRACT Unclassified
20. LIMITATION OF ABSTRACT

UL 